\title{
Efeitos do Controle Aversivo no Contexto de Tratamento Odontopediátrico
}

\author{
Gustavo Sattolo Rolim \\ Antonio Bento Alves de Moraes \\ Universidade Estadual de Campinas \\ Aderson Luiz Costa Jr. \\ Universidade de Brasília
}

\begin{abstract}
RESUMO
O objetivo desse trabalho foi descrever as estratégias empregadas pelo cirurgião-dentista e seus possíveis efeitos na modificação de comportamentos de fuga ou esquiva de crianças em tratamento odontológico. Os participantes foram um dentista e quatro crianças. O procedimento consistiu em oito sessões divididas em duas condições experimentais. Na Primeira Condição, o atendimento era conduzido de acordo com procedimento padrão, exceto pela exigência de não utilização de contenção física. Esta exigência foi suspensa na Segunda Condição. Todas as sessões foram gravadas em vídeo e analisadas seqüencialmente. Os resultados mostraram que crianças, quando expostas à situação odontológica, apresentam diminuição na freqüência de suas respostas de resistência e protesto frente às demandas do tratamento. Frente a não-colaboração das crianças, o dentista utiliza-se da Contenção Física para realizar o tratamento. Os resultados sugerem que a situação odontológica é importante não apenas para a saúde bucal, mas também para a promoção de respostas de enfrentamento de pacientes odontopediátricos expostos a situações que envolvam eventos aversivos. No entanto, do ponto de vista ético e da aprendizagem de novos comportamentos, levanta-se a discussão da relevância do controle aversivo em odontologia.
\end{abstract}

Palavras-chave: psicologia aplicada à odontologia; manejo comportamental; controle aversivo.

\begin{abstract}
Effects of Aversive Control in the Pediatric Dentistry

This study aimed to describe management strategies used by dentists and their possible effect in the modification of escape or avoidance behaviors of children undergoing dental treatment. The participants included four children and one dentist. The procedure consisted of eight sessions separated in two experimental conditions. In the First Condition, the treatment followed regular procedures but physical restraint were used. In the Second Condition, the dentist could decide what management strategy to use including physical restraint. All the sessions were recorded and observed in VT and sequentially analyzed. Results indicated that when children were exposed to dental treatment they presented an overall reduction of resistance and protest responses. However, when allowed, dentists manage uncooperative responses with physical restraint, to maintain the routine of dental procedures. Results suggest that dental treatment is an important situation to promote coping responses of pediatric patients submitted to aversive stimuli. Then from an ethical point of view and that of learning of new repertoires, this paper raises a discussion about the relevance of aversive control in childhood dentistry.

Keywords: psychology applied to dentistry; behavior management strategies; aversive control.
\end{abstract}

As situações em saúde, freqüentemente, estão associadas à dor e/ou desconforto e é papel do profissional lidar com as percepções e sentimentos expressos pelo paciente de modo a reduzir o sofrimento e auxiliá-lo no restabelecimento de sua saúde. Estas habilidades envolvem tanto aspectos técnicos, como a administração de fármacos, como também, ações ativas, como escuta, comunicação e ensino de habilidades, estas muitas vezes não observadas nas rotinas clínicas dos serviços de saúde.

O cuidado em saúde está relacionado a eventos ou experiências percebidas como adversas ou aversivas (dor potencial, desconforto e/ou dor real, por exemplo). Estas se relacionam ao processo de ansiedade 
percebida pelos usuários, que passam a antecipar um sofrimento real ou potencial e evitar a situação. Desta maneira, aponta-se a necessidade da análise dos comportamentos que competem com as respostas de fuga e as respostas emocionais dos indivíduos. Frente às respostas de ansiedade dos usuários, o profissional da saúde (cirurgião-dentista, médico, enfermeiro etc.), pode, com objetivo de proporcionar maior confiança e adesão do usuário, fazer uso de estratégias de manejo de comportamentos. Tais estratégias são caracterizadas por um conjunto de intervenções psicológicas que visam estabelecer situações novas e reforçadoras para o enfrentamento dos tratamentos curativos, odontológico ou não (Rolim, Moraes, César \& Costa Junior, 2004).

As estratégias não coercitivas são, certamente, mais aceitas socialmente (Costa e cols., 2005). No entanto, utilizá-las com crianças temerosas exige tempo e investimento adicional, desde a formação acadêmica até um planejamento pré-sessão. Este envolve que o processo saúde-doença (bucal), as necessidades psicossociais e o comportamento do usuário sejam considerados na avaliação e prognóstico. Sheller (2004), no entanto, observa que o profissional de saúde é valorizado por sua eficiência técnica, não sendo recompensado por gastar tempo no estudo do manejo comportamental de seu paciente.

Observa-se que ainda não há consenso na literatura sobre a utilização e nem sobre os efeitos do controle coercitivo em pesquisas aplicadas à odontologia. As recomendações aparentemente estão relacionadas a uma questão moral, sentimental ou estrutural do evento (instrumento ou técnica empregada) e não derivam de conhecimento efetivo sobre quando, como e quais seriam seus efeitos (Colares \& Richman, 2002; Connick, Palat \& Pugliese, 2000; Newton, Paptel, Shah \& Sturmey, 2004; Pinkham, 1993; Townend, Dimigen \& Fung, 2000).

Nesse sentido, Costa Junior (2002) aponta que um dos principais objetivos da psicologia aplicada à odontologia é disponibilizar estratégias que promovam a aquisição de comportamentos facilitadores do enfrentamento do tratamento, evitando-se a exposição do paciente a contingências aversivas. Para isso, é indispensável compreender as circunstâncias e as variáveis envolvidas no emprego de estratégias coercitivas em saúde. Este estudo descreve e analisa o processo de interação profissional-usuário de serviço, do ponto de vista do controle aversivo envolvido no atendimento de pacientes odontopediátricos.

\section{MÉTODO}

Os participantes deste estudo foram um cirurgiãodentista e quatro crianças, sendo dois meninos e duas meninas (1,2, 3 e 4) com média de 4,5 anos e que apresentavam histórias de não-colaboração em tratamentos odontológicos, verificadas por meio de registros da própria instituição onde os dados foram coletados.

Foram realizadas oito sessões consecutivas de atendimento odontológico, divididas em duas condições experimentais. Na Primeira Condição, referente às quatro primeiras sessões, o profissional era instruído a executar o tratamento sem adotar qualquer estratégia de ameaça verbal ou restrição física. Na Segunda Condição, da $5^{\text {a }}$ a $8^{\text {a }}$ sessão, o cirurgião-dentista tinha autorização para, se fosse necessário, executar o tratamento com emprego da estratégia comportamental "Contenção Física".

Para todas as sessões foram planejados os mesmos procedimentos odontológicos. A única diferença era a possibilidade ou não da utilização da contenção física na segunda condição experimental. Todas as sessões foram registradas em vídeo. As estratégias empregadas pelo cirurgião-dentista para manejo de comportamentos dos pacientes foram categorizadas e registradas em termos de freqüência e conforme as rotinas odontológicas em que ocorriam. As estratégias categorizadas foram obtidas a partir do estudo de Possobon (2003): (a) Diretiva - informações disponibilizadas à criança com objetivo de explicar o que vai ser realizado na sessão de atendimento; (b) Distrativa - ações que visam deslocar a atenção da criança para eventos prazerosos e concorrentes com o tratamento, tais como cantar, conversar e contar história; e (c) Contenção Física - ações do cirurgião-dentista, do auxiliar e do acompanhante que seguram a criança de maneira a restringir seus movimentos e permitir a execução do tratamento.

Os comportamentos dos pacientes também foram categorizados e registrados em termos de freqüência e em função das estratégias empregadas pelo cirurgiãodentista. Para análise de dados, foram destacadas as seguintes categorias de comportamentos não-colaborativos das crianças (Possobon 2003): (a) Resistência respostas motoras que impedem o início do tratamento odontológico; (b) Movimentar-se - respostas motoras, pouco intensas e repetitivas, que não impedem a execução do tratamento; (c) Choro - evocação de sons mais ou menos agudos com seguimento, ou não, de lágrimas. 
As categorias não são mutuamente excludentes, pois as crianças poderiam apresentar Resistência (a) frente algum procedimento e no mesmo momento apresentar a respostas como Choro (c), como também a categoria Movimento Nervoso (b). Ou seja, durante um atendimento odontológico a criança poderia, ao mesmo tempo, resistir a uma contenção realizada pela sua mãe (cuidador segura mãos da criança junto ao peito da mesma e o paciente movimenta e abre as mãos - faz força - que estão impedidas de movimentar-se), ao mesmo tempo em que, movimenta as pernas repetidamente e chora.

\section{RESULTADOS}

Os resultados obtidos são apresentados em figuras de registro cumulativo de respostas dos participantes e do cirurgião-dentista, identificando-se a rotina odontológica a que se refere e a sessão de tratamento odontológico em cada condição experimental (Figuras $1,2,3$ e 4$)$.

No atendimento ao participante 1 , sob a condição experimental 1 (parte superior), observou-se que $o$ tratamento foi executado em todas as rotinas odontológicas, o que caracteriza a colaboração da criança. $\mathrm{Na}$ segunda condição experimental (parte inferior), a estratégia contenção física produziu respostas de fuga por parte da criança na rotina odontológica de preparo cavitário.

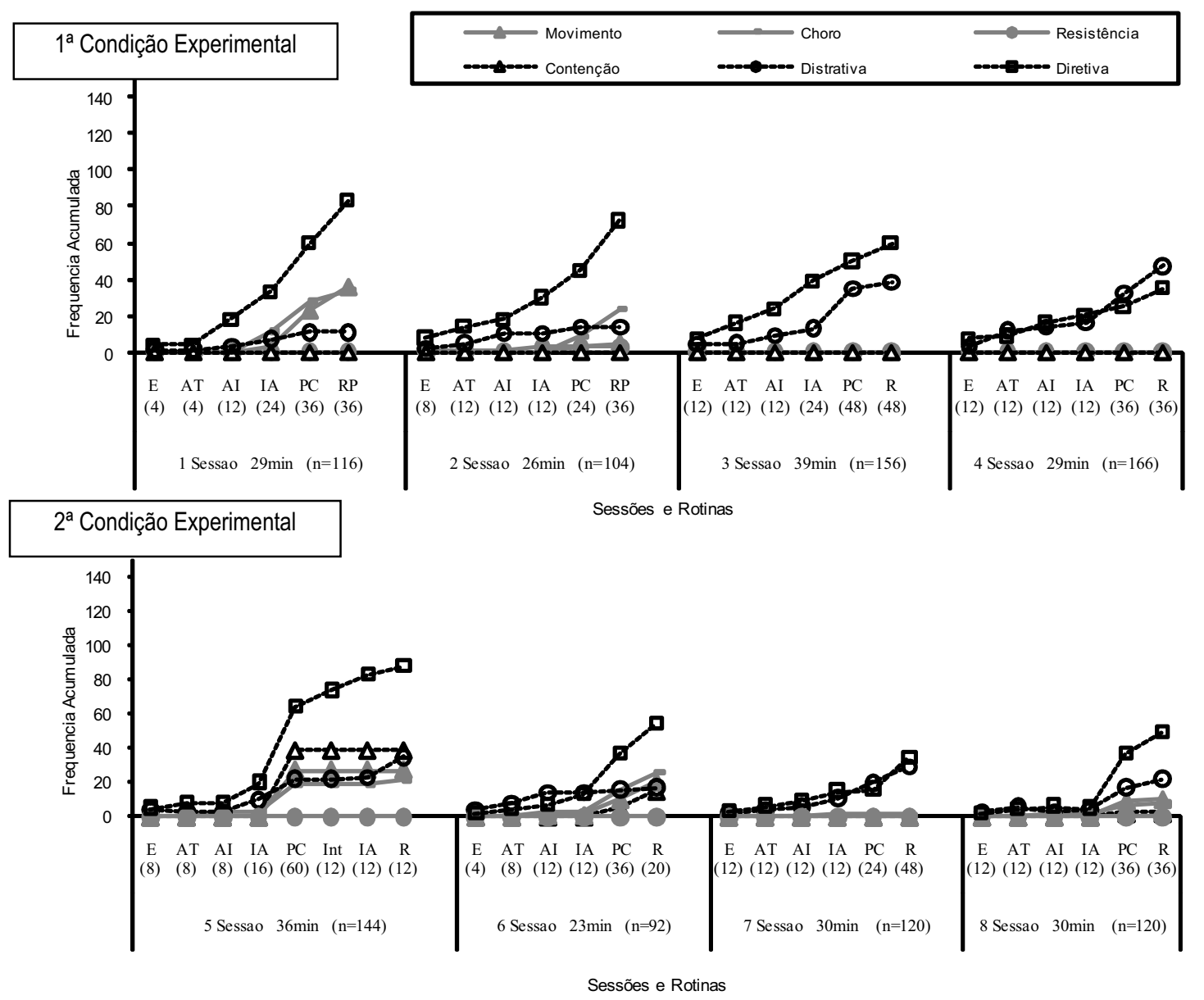

Figura 1. Freqüência acumulada das respostas de P1 e do dentista em cada condição experimental. 
$\mathrm{Na}$ segunda condição experimental, observa-se uma antecipação do cirurgião-dentista ao comportamento categorizado como Movimento Nervoso da criança, isto é, o dentista está de prontidão para executar a contenção física frente a qualquer comportamento físico ou de protesto verbal da criança, independentemente de interferir na execução do tratamento, ou não. Levanta-se a hipótese de que, nesta condição experimental, o contexto (a criança e/ou outras variáveis presentes na situação) adquire proprie- dade aversiva para o dentista; às quais ele responde com o uso da Contenção Física.

Para o participante 2, verificou-se que a rotina específica de Anestesia Infiltrativa estava relacionada às respostas de esquiva e fuga na primeira condição experimental. No momento em que a criança foi contida fisicamente, o tratamento odontológico foi realizado integralmente. Durante esses atendimentos, da quinta à oitava sessões, a criança colaborou, porém, sob protesto verbal.

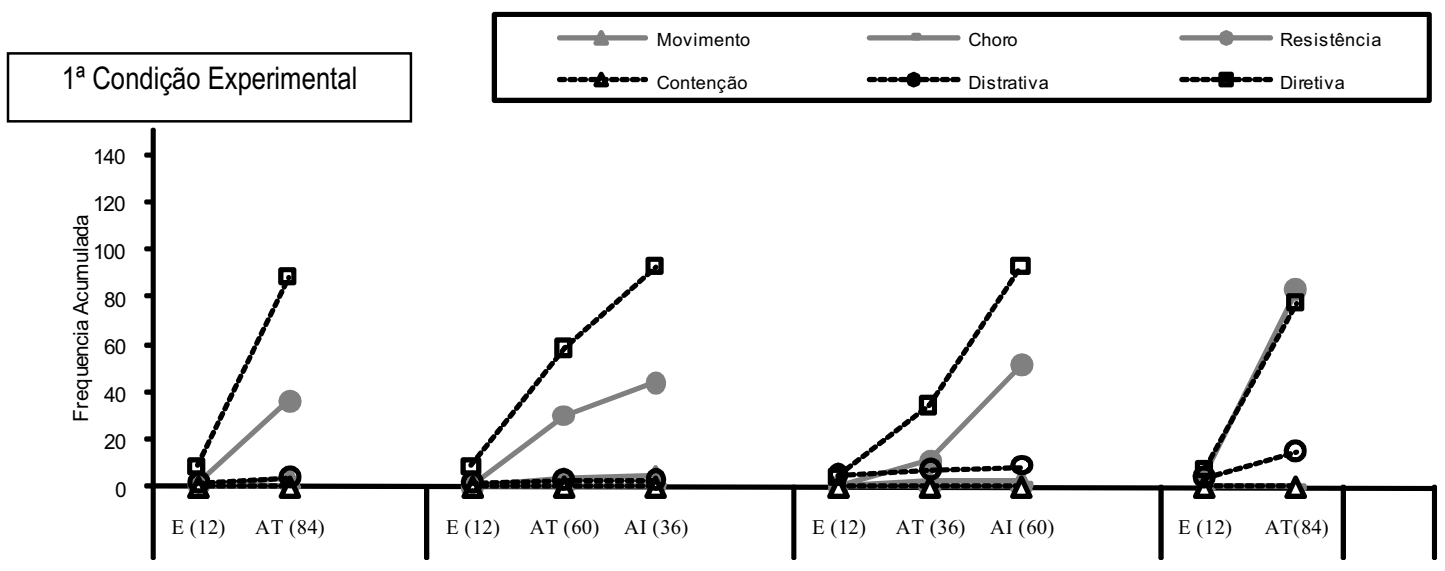

Sessões e Rotinas

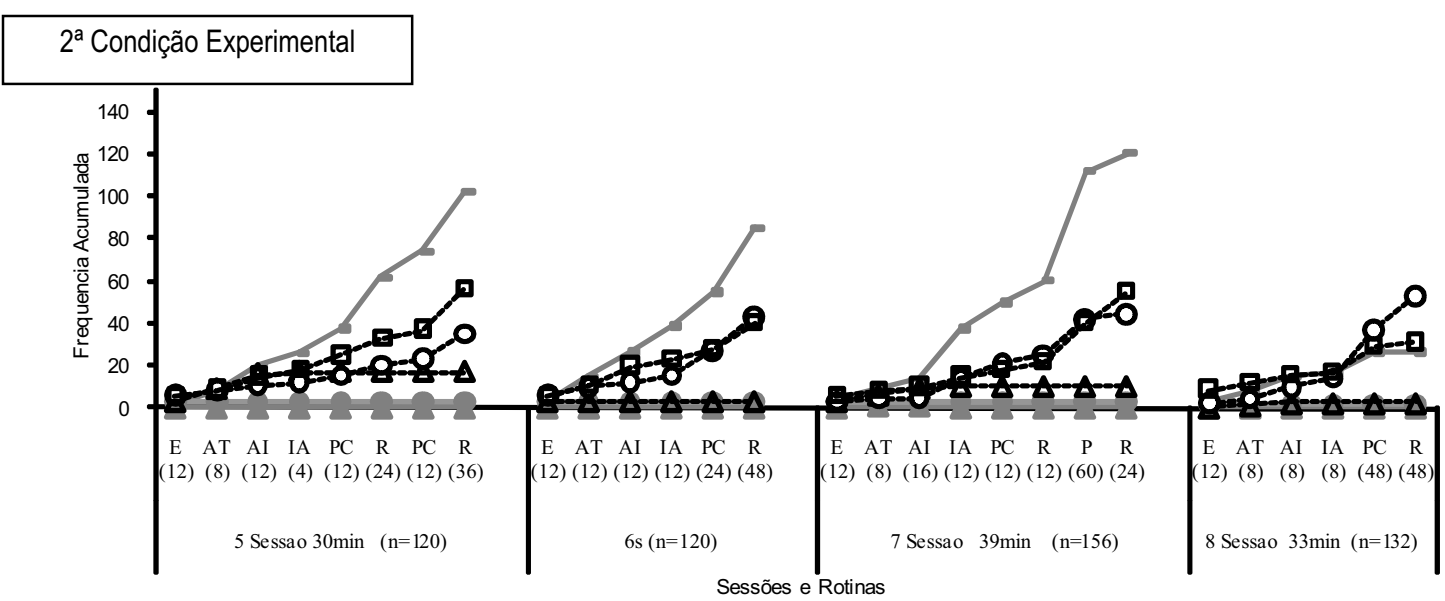

Figura 2. Freqüência acumulada das respostas de $\mathrm{P} 2$ e do dentista em cada condição experimental.

Com relação à escolha da estratégia de Contenção Física para o Participante 2, pode-se dizer que esta foi eficaz, isto é, teve efeito na modificação das respostas de esquiva da criança, que se tornaram menos freqüentes após a realização da técnica, caracterizando um procedimento de punição de respostas. A nãoevocação de respostas de Resistência, embora tenha permitido o tratamento, não foi seguida de respostas de colaboração da criança. Este participante emitiu, em todas as sessões da Segunda Condição, respostas 
de Choro. Tais respostas podem ser interpretadas como esquiva supersticiosa da criança, que não modificam o contexto, mas que são mantidas pelos estímulos da situação. Parece que, par a a criança o chorar produz algum alivio. Respostas de Choro permitem o tratamento e, aparentemente, o dentista não está preparado para modificá-las.
Com relação ao Participante 3, observa-se que não foi possível executar rotinas odontológicas na Primeira Condição Experimental. Na Segunda Condição, a criança foi impedida de evocar respostas que evitavam o tratamento, uma vez que foi submetida ao tratamento odontológico sob contenção física em todas as sessões.
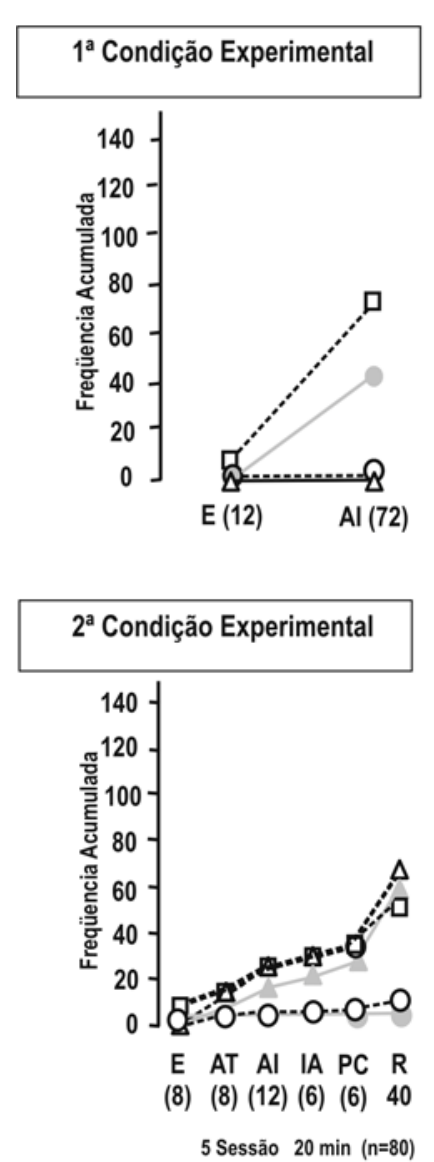
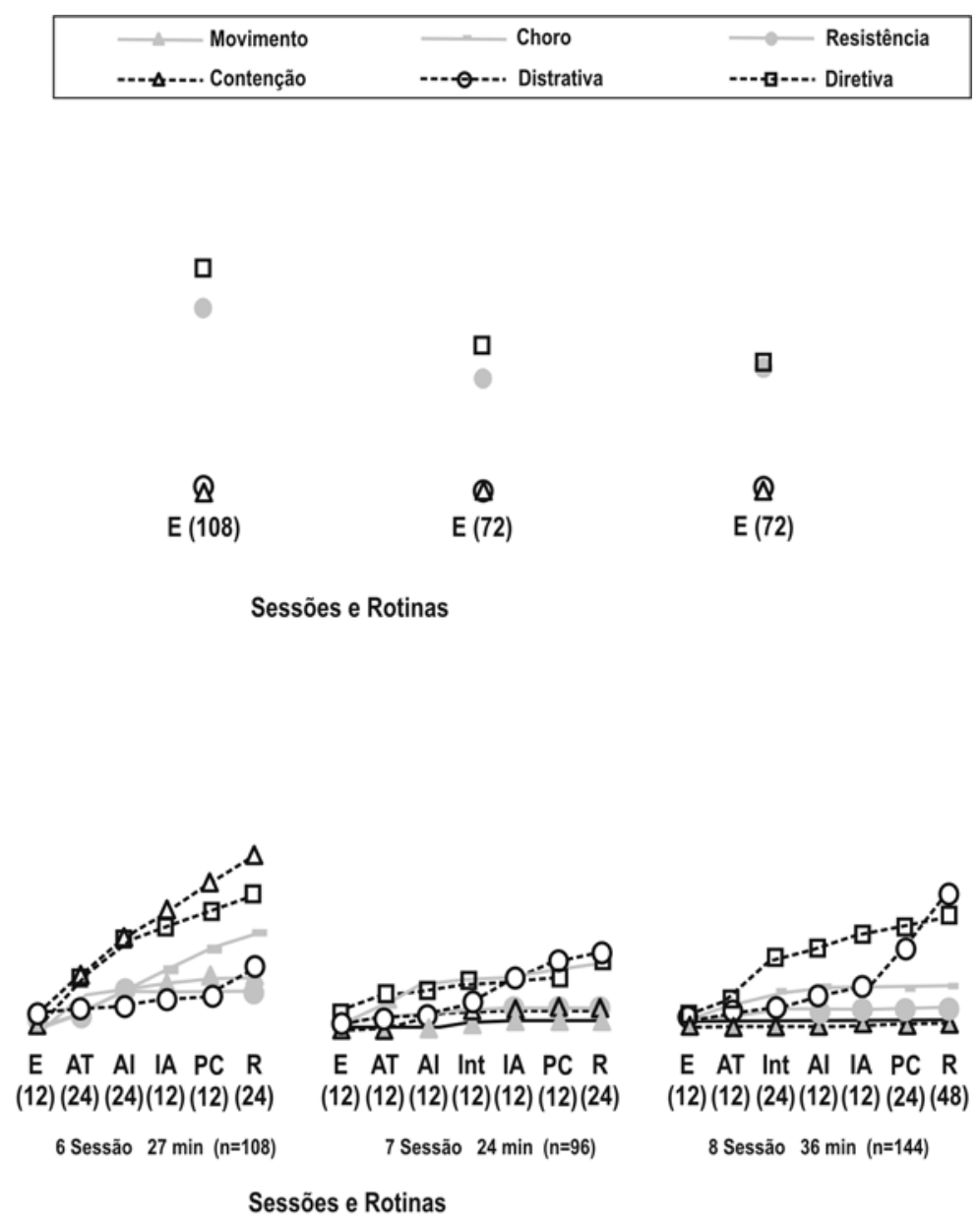

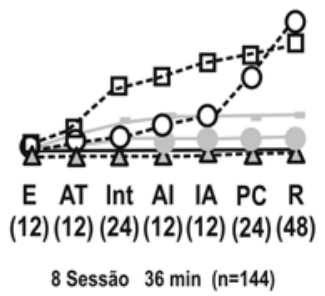

8 Sessão 36 min ( $n=144)$

Figura 3. Freqüencia acumulada das respostas de $\mathrm{P} 3$ e do dentista em cada condição experimental

Aparentemente, os comportamentos de Resistência tornaram-se menos freqüentes, na Primeira Condição Experimental, embora tenham sido eficazes para impedir a realização das rotinas odontológicas. Apesar de não ter sido possível executar qualquer procedimento invasivo, a criança entrou no ambiente odontológico sem emitir respostas como choro ou de afastamento.

Na Segunda Condição Experimental, a criança foi submetida ao tratamento e as respostas de Movimento que adiavam as intervenções não ocorreram em função do uso de Contenção Física. Diante da não colaboração da criança, o profissional utilizou-se da Contenção Física, embora esta estratégia não tenha produzido supressão da não colaboração. Pode-se observar que a Contenção enfraqueceu o responder da criança (Resistência), e que as outras estratégias comportamentais (diretivas e distrativas) não mostraram efetividade na modificação comportamental da criança. 
No atendimento ao Participante 4, verificou-se que na Primeira Condição $\left(1^{\mathrm{a}}\right.$ a $4^{\mathrm{a}}$ sessões - com a vigência da regra "não utilizar contenção física") não foi possível executar o tratamento odontológico. Nas quatro primeiras sessões, a categoria de maior freqüência da criança foi Resistência e do dentista foi Diretiva. Houve também, em menor freqüência, a ocorrência da categoria Distrativa (categoria comportamental do dentista) a partir da rotina Anestesia Tópica da segunda sessão de atendimento.
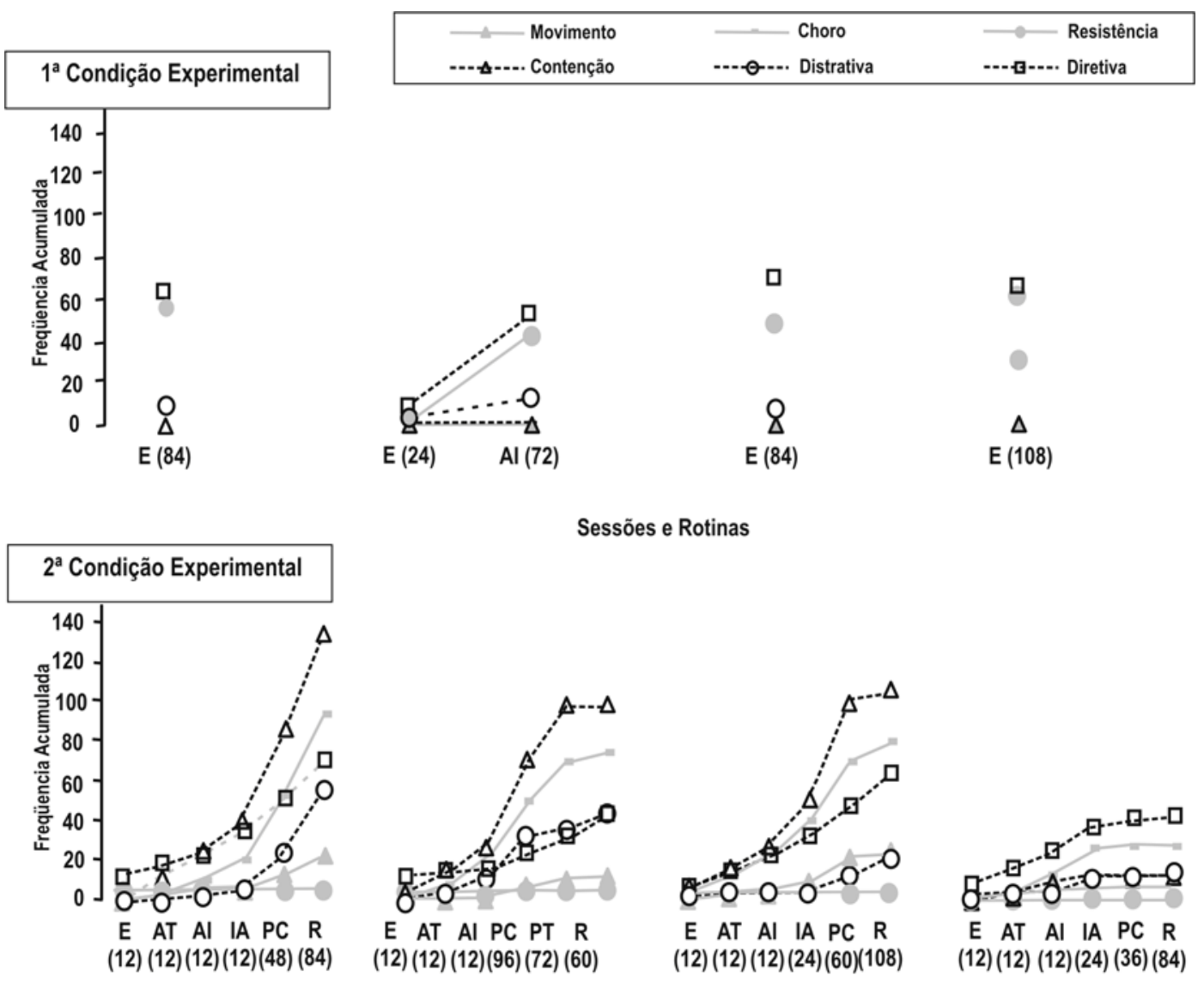

5 Sessão $45 \mathrm{~min}(\mathrm{n}=180)$

6 Sessão $66 \min (n=264)$

7 Sessão $57 \mathrm{~min}(n=228)$

8 Sessão $45 \mathrm{~min}(n=180)$

Sessões e Rotinas

Figura 4. Freqüencia acumulada das respostas de $\mathrm{P} 4$ e do dentista em cada condição experimental

Na Segunda Condição (quinta a oitava sessões), o atendimento foi realizado independentemente dos comportamentos emitidos pela criança. Observou-se que as respostas de Resistência da criança diminuíram em freqüência até o final do tratamento. Nestas sessões, o paciente foi submetido compulsoriamente ao tratamento com a realização da Contenção Física. Pode-se observar que a criança foi restrita durante todas as rotinas odontológicas das três sessões iniciais da Segunda Condição e que essa técnica ou quaisquer outras respostas do cirurgião não modificaram os comportamentos de não colaboração. Destaca-se, assim, que os comportamentos evitativos não se mostraram sensíveis as estratégias empregadas.

\section{DISCUSSÃO}

De modo geral, pode-se considerar que em atendimentos de saúde, que envolvem procedimentos clínicos invasivos, a condição de tratamento torna-se aversiva para díade - profissional e usuário - e que esta é agravada com o uso de contenção física. Para todos os participantes, a estratégia restritiva, foi um recurso 
aversivo. Ou seja a contenção física produziu contracontrole (P1), supressão das respostas de resistência (P2 - processo de punição), e variabilidade e diminuição das respostas de resistência (P3 e P4 - processo de extinção)

Aparentemente, o repertório comportamental do dentista mostra-se limitado diante de crianças que apresentam níveis altos de não colaboração. É possível observar que frente a comportamentos que interrompem o tratamento, atrapalham as ações clínicas ou sinalizam sofrimento, o profissional tende a responder com o uso de contenção; esta seria uma resposta estereotipada do profissional diante de padrões de comportamento percebidos como aversivos. Quando a restrição não é possível, como na Primeira Condição, então o dentista passa a empregar outras estratégias, entre as quais as Diretivas tendem a ocorrer com maior freqüência. $\mathrm{O}$ dentista que não pode utilizar contenção física, direciona e instrui a criança sobre os procedimentos e também persuade o paciente sobre as necessidades do tratamento. Essas verbalizações, neste estudo, mostraram-se ineficazes para produzir comportamentos de aceitação do tratamento.

Com relação ao responder da criança, os dados sugerem que a Contenção não foi uma estratégia planejada. O emprego da estratégia para o Participante 1, produziu contracontrole. Um efeito indesejável, ocasionado pela prontidão do profissional em conter. $\mathrm{Ou}$ seja, um equívoco na escolha da estratégia comportamental. Sem a regra de proibição à contenção física, a resposta imediata do profissional é terminar com a estimulação aversiva (que poderia ser uma estimulação aversiva para ele profissional). Os dados sugerem que a situação odontológica é aversiva para o dentista e que a contenção resulta em reforçamento negativo para seus comportamentos.

Em outros casos, as respostas de esquiva ou fuga diminuem de freqüência de sessão para sessão, o que sugere um processo de extinção (Figuras 3 e 4), embora a criança esteja sendo submetida a apresentação de estímulos aversivos. Destaca-se que a estratégia Contenção Física apenas permitiu a realização do atendimento, porém com alta freqüência de comportamentos que sugerem desconforto, protesto e sofrimento. A estratégia foi eficaz para a modificação comportamental a longo prazo. No entanto, sugere-se que frente a situação como estas outras estratégias poderiam ter sido empregadas (fuga contingente - Allen e Stokes, 1987; modelação - Stokes e Kennedy, 1980).
Com relação ao Participante 2, respostas de esquiva foram punidas, ou seja, a Contenção Física ocasionou a diminuição abrupta no responder. A criança deixa de se movimentar, porém com a manutenção da situação estimuladora (tratamento odontológico), ela protesta verbalmente. Frente a esta condição, o profissional possui pouco repertório (O profissional não tenta ou não precisa acalmar a criança).

Pode-se sugerir que as crianças que não permitem o tratamento, ou seja, a realização de rotinas não invasivas, deveriam passar por uma dessensibilização ou por um treinamento que permitisse a adaptação às exigências do tratamento. A experiência clínica tem mostrado que a exposição gradual ao tratamento de crianças altamente temerosas fortalece respostas de esquiva e solicita a realização de inúmeras sessões de atendimento o que aumenta a aversividade da situação para a criança e para o dentista (Possobon, 2003).

A importância de analisar a situação de interação está relacionada às práticas clínicas e a busca de opções de intervenção. A utilização de estratégias deve ser planejada, assim como, o atendimento clínico é sistematicamente orientado. O profissional deve orientar sua prática em relação a demanda clínica e comportamental. Se o profissional da saúde não observar, não analisar e não refletir sobre sua intervenção, sua prática negligenciará a participação dos usuários, assim como, o atendimento de suas necessidades.

Desconhecer as variáveis que estão relacionadas à situação de atendimento, possivelmente, mantém o repertório dos profissionais. Diante de uma criança altamente não colaboradora, estes emitem respostas que eliminam os eventos aversivos. A Contenção Física, foi uma resposta generalizada, reforçada negativamente e reforçada positivamente por permitir a realização do tratamento odontológico. Desta maneira, sugere-se que o uso de contenção física deva ser superado e que estratégias outras devam auxiliar as crianças a enfrentarem esta situação.

\section{REFERÊNCIAS}

Allen, K. D., \& Stokes, T. F. (1987). Use of escape and reward in the management of young children during dental treatment. Journal of Applied Behavior Analysis, 20, 381-90.

Colares, V., \& Richman, L. (2002). Factors associated with uncooperative behavior by Brazilian preschool children in the dental office. Journal of Dentistry for Children, 69, 87-91.

Connick, C., Palat, M., \& Pugliese, S. (2000). The appropriate use of physical restraint: Considerations. Journal of Dentistry for Children, 76, 256-262.

Costa Junior, A. L. (2002). Psicologia aplicada à odontologia: Uma introdução. Estudos e Pesquisas em Psicologia, 2, 67-77. 
Costa, M. E. P. R., Charlier, S. C., Costa, V. R., Charlier, F. C., Ferreira, F. T. S. C., \& Neves, M. L. (2005). Reações dos pais, odontopediatras e psicólogos às técnicas de controle de comportamento utilizadas em odontopediatria. Trabalho apresentado no $12^{\circ}$ Congresso Nacional da ABOPREV: Tecnologia e Humanização na Promoção da Saúde, Rio de Janeiro.

Newton, J. T., Paptel, H., Shah, S., \& Sturmey, P. (2004). Attitudes towards the use of hand over mouth (HOM) and physical restraint amongst paediatrtric specialist practioners in UK. International Journal of Paediatric Dentistry, 14, 111-117.

Pinkham, J. R. (1993). The roles of requests and promises in child patient management. Journal of Dentistry for Children, 60, 169-174.

Possobon, R. F. (2003). Efeitos do diazepam sobre os comportamentos não colaborativos de crianças em atendimento odontológico. Tese de doutorado não-publicada, Universidade Estadual de Campinas, Piracicaba, SP.
Rolim, G. S., Moraes, A. B. A., César, J., \& Costa Junior, A. L. (2004). Análise do comportamento do odontólogo no contexto de atendimento infantil. Estudos de Psicologia, 9, 533-541.

Stokes, T. F., \& Kennedy, S. H. (1980) Reducing child uncooperative behavior during dental treatment through modeling and reinforcement. Journal of Applied Behavior Analysis, 13, 41-49.

Townend, E., Dimigen, G., \& Fung, D. (2000). A clinical study of child dental anxiety. Behaviour Research and Therapy, 38, 3146 .

Recebido: $16 / 05 / 2007$

Última revisão: $06 / 05 / 2008$

Aceite final: $25 / 05 / 2008$

\section{Sobre os autores:}

Gustavo Sattolo Rolim: Doutorando do Programa de Pós-Graduação da Saúde da Criança e do Adolescente da FCM/UNICAMP. Endereço eletrônico: gurolim@gmail.com.

Antonio Bento Alves de Moraes: Prof. Dr. - FOP/UNICAMP. Endereço eletrônico: abento@fop.unicamp.br.

Aderson Luiz Costa Jr.: Prof. Dr. - Instituto de Psicologia - UnB. Endereço eletrônico: aderson@unb.br.

Endereço para correspondência: Universidade Estadual de Campinas - Faculdade de Odontologia de Piracicaba - Av. Limeira, 901 13414-903 - Piracicaba - SP. 